\title{
On the Rarity of Quasinormal Subgroups
}

\author{
John Cossey (*) - Stewart Stonehewer (**)
}

\begin{abstract}
For each prime $p$ and positive integer $n$, Berger and Gross have defined a finite $p$-group $G=H X$, where $H$ is a core-free quasinormal subgroup of exponent $p^{n-1}$ and $X$ is a cyclic subgroup of order $p^{n}$. These groups are universal in the sense that any other finite $p$-group, with a similar factorisation into subgroups with the same properties, embeds in $G$. In our search for quasinormal subgroups of finite $p$-groups, we have discovered that these groups $G$ have remarkably few of them. Indeed when $p$ is odd, those lying in $H$ can have exponent only $p, p^{n-2}$ or $p^{n-1}$. Those of exponent $p$ are nested and they all lie in each of those of exponent $p^{n-2}$ and $p^{n-1}$.
\end{abstract}

\section{Introduction.}

A subgroup $Q$ of a group $G$, such that $Q H=H Q$ for all subgroups $H$ of $G$, is said to be quasinormal (sometimes permutable) in $G$ and we write Q qn G. The concept was introduced by Ore in 1937 (see[6]) and in [7] he proved that, in finite groups, quasinormal subgroups are always subnormal. He also proved that quasinormal subgroups are modular. Recall that a subgroup $M$ of a group $G$ is modular if, for all subgroups $X$ and $Y$ of $G$,

$$
\langle X, M\rangle \cap Y=\langle X, M \cap Y\rangle \text { if } X \leqslant Y
$$

and

$$
\langle X, M\rangle \cap Y=\langle X \cap Y, M\rangle \text { if } M \leqslant Y .
$$

Indeed a subgroup $Q$ of a finite group $G$ is quasinormal in $G$ if and only if $Q$ is modular and subnormal in $G$. Thus the concepts of modularity and

(*) Indirizzo dell'A.: Mathematics Department, School of Mathematical Sciences, Australian National University, Canberra, ACT 0200.

E-mail: John.Cossey@anu.edu.au

(**) Indirizzo dell'A.: Mathematics Institute, University of Warwick, Coventry CV4 7AL, England.

E-mail: S.E.Stonehewer@warwick.ac.uk 
quasinormality coincide in finite $p$-groups. In 1973 Maier and Schmid proved in [5] that if $G$ is a finite group and $Q q n G$ with $Q$ core-free, i.e. $Q_{G}=1$, then $Q$ lies in the hypercentre of $G$. It follows fairly easily from this that the complexities of the embedding of $Q$ in $G$ reduce to the case where $G$ is a $p$-group, i.e. where $Q$ is a modular subgroup. Bearing in mind that modularity is a property invariant under subgroup lattice isomorphisms, the quasinormal subgroups of a finite $p$-group $G$ are surely relevant when the structure of $G$ is approached via its lattice of subgroups.

It was shown in [10] that given $Q q n G$ and $Q$ abelian, then $Q^{n} q n G$, provided $n$ is odd or divisible by 4 (even when $G$ is infinite). Apart from this, very little appears to be known about which subgroups of $Q$ are also quasinormal in $G$.

Clearly $Q q n G$ if and only if $Q X$ is a subgroup for each cyclic subgroup $X$ of $G$. Thus the situation $G=Q X$ is an obvious starting point for investigations. The case when $G$ here is a finite $p$-group (for $p$ an odd prime), and $Q$ is an abelian quasinormal subgroup of $G$ (with $X$ cyclic), is studied in [2]. It is shown that there are two composition series of $G$ passing through $Q$, all the members of which are quasinormal in $G$. The attempt to remove the hypothesis that $Q$ is abelian has produced the present work. We have discovered a situation very far removed from the abelian case. In fact there is a family of finite $p$-groups $G$ (for each odd prime $p$ ) with $Q$ qn $G$ such that

(i) there are subgroups $L \triangleleft M \leqslant Q$, with $L, M$ qn $G$;

(ii) $G$ has no quasinormal subgroup strictly between $L$ and $M$;

(iii) $L$ has exponent $p$ and $M$ has exponent $p^{m}$; and

(iv) $m$ and the nilpotency class (even derived length) of $M / L$ can be greater than any given positive integer.

These groups $G$ were constructed by Berger and Gross in [1] and they are defined as follows. Let $n$ be a positive integer and $p$ be an odd prime. We define $\Gamma_{n}$ to be the additive group Z of integers modulo $p^{n} Z$. Let $x_{n}$ be the permutation of $\Gamma_{n}$ given by

$$
x_{n}: p^{n} \text { Z }+\ell \mapsto p^{n} \text { Z }+\ell+1 .
$$

Then $x_{n}$ belongs to the symmetric group of degree $p^{n}$. For $0 \leqslant m \leqslant n$, define

$$
x_{n, m}=x_{n}^{p^{n-m}}, \text { of order } p^{m} .
$$

Let $X_{n}=\left\langle x_{n}\right\rangle$, a cyclic group of order $p^{n}$; and let $X_{n, m}=\left\langle x_{n, m}\right\rangle$, the subgroup of order $p^{m}$. Define $\Delta_{n, m}$ to be the set of elements in the additive 
group $\Gamma_{n}$ of order $p^{m}$. So

$$
\Delta_{n, m}=\left\{\lambda p^{n-m} \mid 0 \leqslant \lambda \leqslant p^{m}-1, p \nmid \lambda\right\} \text { for } 1 \leqslant m \leqslant n
$$

and $\Delta_{n, 0}=\{0\}$. Then $\left|\Delta_{n, m}\right|=p^{m-1}(p-1)$ for $1 \leqslant m \leqslant n$. The permutation $x_{n, m}$ shifts by $p^{n-m}$ and fixes the set $\Delta_{n, m+1}$, i.e. the set of $\lambda p^{n-m-1}(p \nmid \lambda)$ between 0 and $p^{n}-1$, for $0 \leqslant m \leqslant n-1$. Thus $x_{n, m}$ acting on the set $\Delta_{n, m+1}$ (of cardinality $p^{m}(p-1)$ ) is the product of $p-1$ disjoint cycles $\pi_{n, m, i}$ of length $p^{m}, 1 \leqslant i \leqslant p-1$, each cycle adding $p^{n-m}$. So

$$
\pi_{n, m, i}=\left(i p^{n-m-1}, i p^{n-m-1}+p^{n-m}, \ldots, i p^{n-m-1}+\left(p^{m}-1\right) p^{n-m}\right) .
$$

Now for $0 \leqslant m \leqslant n-1$, define

$$
A_{n, m}=\left\{\prod_{i=1}^{p-1} \pi_{n, m, i}^{c_{i}} \mid \sum_{i=1}^{p-1} c_{i}=0\right\} .
$$

Each permutation in this set is a product of disjoint cycles, each containing an integer of the form $\lambda p^{n-m-1}(p \nmid \lambda)$ and shifting by a multiple of $p^{n-m}$. In fact $A_{n, m}$ is an abelian group isomorphic to the direct product of $p-2$ cyclic groups of order $p^{m}$. (See [1], Lemma 3.1 (1).) Its elements fix every integer not in $\Delta_{n, m+1}$.

Berger and Gross define the group

$$
G_{n}=\left\langle x_{n}, A_{n, m} \mid 0 \leqslant m \leqslant n-1\right\rangle,
$$

and $H_{n}$ to be the stabiliser of 0 in $G_{n}$. So

$$
G_{n}=H_{n} X_{n},
$$

a finite $p$-group. Also $H_{n}$ has exponent $p^{n-1}$. The main result of [1] is:-

THEOREM. The subgroup $H_{n}$ is core-free and quasinormal in $G_{n}$. Moreover, if $H^{*}$ is a core-free quasinormal subgroup of a finite p-group $G^{*}=H^{*}\left\langle x^{*}\right\rangle$, where $\left\langle x^{*}\right\rangle$ is a cyclic group of order $p^{n}$, then there is a unique embedding $\psi$ of $G^{*}$ in $G_{n}$ such that $\psi\left(x^{*}\right)=x_{n}$ and $\psi\left(H^{*}\right) \leqslant H_{n}$.

Our main results concern the quasinormal subgroups of $G_{n}$ that lie in $H_{n}$. In the Berger-Gross Theorem above, the prime $p$ is arbitrary. However, the case $p=2$ requires much additional analysis. Accordingly in all our work here we assume that

the prime $p$ is odd.

We shall prove 
THEOREM 1. Let $n \geqslant 2$ and let $Q$ be a non-trivial quasinormal subgroup of $G_{n}$ lying in $H_{n}$ of exponent $p^{k}$. Then one of the following holds:-

(i) $k=1$;

(ii) $n \geqslant 4$ and $k=n-2$;

(iii) $n \geqslant 3$ and $k=n-1$.

Moreover for $n \geqslant 4$, the quasinormal subgroups of exponent $p$ are nested and are contained in all those of exponent $p^{n-2}$ and exponent $p^{n-1}$. Thus there is a maximal quasinormal subgroup $L$ of exponent $p$ and a quasinormal subgroup $M$ of exponent $p^{n-2}$ such that $L \leqslant M$ and

there are no quasinormal subgroups of $G_{n}$ in the interval $[M / L]$.

Clearly $L \triangleleft M$. Also $M / L$ has exponent $p^{n-3}$ and there is no bound to its derived length as $n$ increases. (See [9].)

In Theorem 2, we find all the quasinormal subgroups of $G_{n}$ lying in $H_{n}$ and of exponent $p$. Theorem 3 gives first some necessary conditions for subgroups of $G_{n}$, lying in $H_{n}$ and of exponent $p^{n-1}$, to be quasinormal in $G_{n}$; then necessary and sufficient conditions for certain subgroups of exponent $p^{n-1}$ to be quasinormal in $G_{n}$. Theorem 4 is similar, dealing with subgroups of exponent $p^{n-2}$. Finally in Theorem 5 we show that there are no quasinormal subgroups of $G_{n}(n \geqslant 5)$, lying in $H_{n}$ and of exponent $p^{k}$, for $2 \leqslant k \leqslant n-3$. Theorem 1 follows from these results.

We use standard notation for familiar concepts, together with that above introduced by Berger and Gross. Also we switch from multiplicative to additive notation for computation with modules over groups. In this connection there is a considerable amount of calculation involved and the following list should be helpful.

$(\alpha)_{\beta, \gamma}$ : the cyclic permutation containing $\alpha$, shifting by $\beta$, of length $\gamma$. Here $\alpha$ is a non-negative integer and $\beta$ and $\gamma$ are positive integers such that $\beta \gamma=p^{n}$.

$\left\{b_{i, k} \mid r_{k} \geqslant i \geqslant 1\right\}$ : a basis (modulo $\Omega_{k-1}\left(H_{n}\right)$ ) of the elementary abelian group $\Omega_{k}\left(H_{n}\right) / \Omega_{k-1}\left(H_{n}\right)$.

$B_{\ell_{k}, k}$ : the subgroup $\left\langle b_{i, k} \mid \ell_{k} \geqslant i \geqslant 1\right\rangle$.

$B_{k}$ : the subgroup $B_{r_{k}, k}$.

$C_{k}$ : the centraliser in $G_{n}$ of the element $x_{n, k}$, i.e. $C_{G_{n}}\left(x_{n, k}\right)$, chiefly when $k=2$.

$r_{k}$ : the rank of the elementary abelian group $\Omega_{k}\left(H_{n}\right) / \Omega_{k-1}\left(H_{n}\right)$, for $1 \leqslant k \leqslant n-1$.

$X_{n}$ : the cyclic group $\left\langle x_{n}\right\rangle$ of order $p^{n}$. 
$x_{n, m}$ : the element $x_{n}^{p^{n-m}}, 0 \leqslant m \leqslant n$.

$X_{n, m}$ : the cyclic subgroup $\left\langle x_{n, m}\right\rangle$ of order $p^{m}$.

$\left\{w_{i} \mid r_{1} \geqslant i \geqslant 1\right\}$ : a basis of the elementary abelian group $\Omega_{1}\left(H_{n}\right)$, and $w_{i}=b_{1, i}$.

$W_{j}$ : the subgroup $\left\langle w_{i} \mid j \geqslant i \geqslant 1\right\rangle\left(=B_{j, 1}\right)$.

$Z_{p^{m}}$ : a cyclic group of order $p^{m}$.

$\Omega_{k}(G)$ : the subgroup of the finite $p$-group $G$ generated by the elements of order at most $p^{k}$.

\section{Description of the groups $G_{n}$.}

It is shown in [1], Lemma 3.14, that

$$
\Omega_{k}\left(G_{n}\right)=\left\langle x_{n}^{-i} A_{n, k} x_{n}^{i} \mid i \geqslant 0\right\rangle \Omega_{k-1}\left(G_{n}\right),
$$

for $1 \leqslant k \leqslant n-1$. Also

$$
\Omega_{k}\left(G_{n}\right) / \Omega_{k-1}\left(G_{n}\right) \text { is elementary abelian of rank } p^{n-k-1}(p-1) .
$$

We see this as follows. By [1], Lemma 3.3, if $n \geqslant 2$, then there is an epimorphism $\tau_{n}: G_{n} \rightarrow G_{n-1}$, viz. $g \mapsto \tau_{n}(g)$ where

$$
\tau_{n}(g): a \bmod p^{n-1} Z_{s} \mapsto b \bmod p^{n-1} Z_{s}
$$

if

$$
g: a \bmod p^{n} Z_{s} \mapsto b \bmod p^{n} Z_{1}
$$

for all integers $a, b$ and $g \in G_{n}$. In particular $\tau_{n}$ maps $H_{n}$ onto $H_{n-1}$ and $x_{n}$ to $x_{n-1}$. The kernel of $\tau_{n}$ is elementary abelian, by [1], Lemma 3.3 (9), and it is precisely $\Omega_{1}\left(G_{n}\right)$, by [1], Lemma 3.10. Also $\left|G_{n}\right|=p^{p^{n-1}}$, by [1], Corollary 3.15. Thus

$$
\Omega_{1}\left(G_{n}\right) \text { is elementary abelian of } \operatorname{rank} p^{n-1}-p^{n-2}=p^{n-2}(p-1) .
$$

Applying $\tau_{n}$ repeatedly for decreasing values of $n$, we see that

$$
G_{n} / \Omega_{k-1}\left(G_{n}\right) \cong G_{n-k+1},
$$

for $2 \leqslant k \leqslant n ;$ and

$$
\Omega_{1}\left(G_{n} / \Omega_{k-1}\left(G_{n}\right)\right)=\Omega_{k}\left(G_{n}\right) / \Omega_{k-1}\left(G_{n}\right) \cong \Omega_{1}\left(G_{n-k+1}\right),
$$

this last subgroup being elementary abelian of rank $p^{n-k-1}(p-1)$, proving (2). We shall use (4) and (5) repeatedly throughout our work. 
By [1], Lemma 3.10,

$$
\Omega_{1}\left(G_{n}\right)=\Omega_{1}\left(H_{n}\right) X_{n, 1} .
$$

Also, by (1),

$$
\Omega_{1}\left(G_{n}\right)=\left\langle x_{n}^{-i} A_{n, 1} x_{n}^{i} \mid i \geqslant 0\right\rangle,
$$

an elementary abelian group of $\operatorname{rank} p^{n-2}(p-1)$, by (2). By [1], Lemma $3.2(4)$, $x_{n, 1}$ lies in the centre of $G_{n}$.

Let

$$
r_{1}=p^{n-2}(p-1)-1=\operatorname{rank} \text { of } \Omega_{1}\left(H_{n}\right),
$$

for $n \geqslant 2$. Recall that $H_{n}$ is core-free in $G_{n}$ and so $\Omega_{1}\left(G_{n}\right)$ is an indecomposable $X_{n}$-module. We find a canonical basis for $\Omega_{1}\left(G_{n}\right)$ as follows. Define

$$
w_{r_{1}}=x_{n}^{p^{n-2}-1}\left(\pi_{n, 1,1}^{-1} \pi_{n, 1,2}\right) x_{n}^{-\left(p^{n-2}-1\right)} \in \Omega_{1}\left(G_{n}\right) .
$$

We introduce the following shorthand notation for cyclic permutations of integers modulo $p^{n} Z$ which just add a fixed integer:-

$$
(\alpha)_{\beta, \gamma}=(\alpha, \alpha+\beta, \alpha+2 \beta, \ldots, \alpha+(\gamma-1) \beta),
$$

where $\beta \gamma=p^{n}$. Then

$$
\pi_{n, 1,1}^{-1} \pi_{n, 1,2}=\left(p^{n-2}\right)_{p^{n-1}, p}^{-1}\left(2 p^{n-2}\right)_{p^{n-1}, p} \in A_{n, 1} .
$$

Note that the two cycles here are disjoint, coming from different orbits of $x_{n, 1}$. Also

$$
w_{r_{1}}=(1)_{p^{n-1}, p}^{-1}\left(1+p^{n-2}\right)_{p^{n-1}, p},
$$

where again the two cycles are disjoint. Therefore $w_{r_{1}}$ fixes 0 and so $w_{r_{1}} \in \Omega_{1}\left(H_{n}\right)$.

For $r_{1}-1 \geqslant i \geqslant 0$, define

$$
w_{i}=\left[w_{i+1}, x_{n}\right]
$$

inductively. Then dropping the suffixes in (8) for the moment and writing $r=r_{1}$ and $x=x_{n}$, we have $w_{r-1}=(1)\left(1+p^{n-2}\right)^{-1}(2)^{-1}\left(2+p^{n-2}\right)=(1)(2)^{-1}\left(1+p^{n-2}\right)^{-1}\left(2+p^{n-2}\right)$, $w_{r-2}=(1)^{-1}(2)^{2}(3)^{-1}\left(1+p^{n-2}\right)\left(2+p^{n-2}\right)^{-2}\left(3+p^{n-2}\right)$, 
$w_{r-j}=(1)^{(-1)^{j+1}}(2)^{?} \ldots\left(j+1+p^{n-2}\right)$

$w_{1}=(1)^{-1}(2)^{?} \ldots\left(p^{n-1}-1\right)$,

$w_{0}=(1)(2)^{?} \ldots\left(p^{n-1}\right)$.

Any 2 cycles above are either the same or disjoint. Hence they commute. Also $w_{i}$, for $r_{1}(=r) \geqslant i \geqslant 1$, fixes 0 and so belongs to $\Omega_{1}\left(H_{n}\right)$. Clearly $w_{r_{1}}, w_{r_{1}-1}, \ldots, w_{0}$ are linearly independent as elements of the module $\Omega_{1}\left(G_{n}\right)$, since each contains a cycle that does not occur in its predecessors. The rank of $\Omega_{1}\left(G_{n}\right)$ is $r_{1}+1$, by (2). Therefore

$$
\left\{w_{i} \mid r_{1} \geqslant i \geqslant 1\right\} \text { is a basis for } \Omega_{1}\left(H_{n}\right)
$$

and

$$
\left\{w_{i} \mid r_{1} \geqslant i \geqslant 0\right\} \text { is a basis for } \Omega_{1}\left(G_{n}\right) .
$$

Clearly $\Omega_{1}\left(H_{n}\right)$ is an indecomposable $X_{n}$-module. For if not, then the centraliser of $X_{n}$ in $G_{n}$ is not cyclic and therefore intersects $\Omega_{1}\left(H_{n}\right)$ non-trivially. Then the normal closure of this intersection in $G_{n}$ would lie in $H_{n}$, giving a contradiction. Also we must have $w_{0} \in\left\langle x_{n, 1}\right\rangle$ and $x_{n, 1}=(1)(2) \ldots,\left(p^{n-1}\right)$, with cycles of length $p$, shifting by $p^{n-1}$. Thus

$$
w_{0}=x_{n, 1}=x_{n}^{p^{n-1}} \text {. }
$$

Note. For any $i, j$, using additive module notation, we have

$$
\left[w_{i}, x_{n}^{p^{j}}\right]=w_{i}\left(x_{n}^{p^{j}}-1\right)=w_{i}\left(x_{n}-1\right)^{p^{j}}=w_{i-p^{j}} .
$$

Here $w_{i}$, for $i<0$, is taken to be 1 .

Next, for $2 \leqslant k \leqslant n-1$, we find a basis for $\Omega_{k}\left(G_{n}\right) / \Omega_{k-1}\left(G_{n}\right)$ as indecomposable $X_{n}$-module of rank

$$
p^{n-k-1}(p-1)=r_{k}+1,
$$

say (see (2)). Notation will necessarily becomes more complicated. By [1], Corollary 3.11,

$$
\Omega_{k}\left(G_{n}\right)=\Omega_{k}\left(H_{n}\right) X_{n, k} .
$$

Thus by analogy with (7), define

$$
b_{r_{k}, k}=x_{n}^{p^{n-k-1}}\left(\pi_{n, k, 1}^{-1} \pi_{n, k, 2}\right) x_{n}^{-\left(p^{n-k-1}-1\right)}
$$


for $1 \leqslant k \leqslant n-1$. Then using the notation (8), we have

$$
\pi_{n, k, 1}^{-1} \pi_{n, k, 2}=\left(p^{n-k-1}\right)_{p^{n-k}, p^{k}}^{-1}\left(2 p^{n-k-1}\right)_{p^{n-k}, p^{k}} \in A_{n, k}
$$

and

$$
b_{r_{k}, k}=(1)_{p^{n-k}, p^{k}}^{-1}\left(1+p^{n-k-1}\right)_{p^{n-k}, p^{k}} .
$$

Since $b_{r_{k}, k}$ fixes $0 \in \Gamma_{n}$, it belongs to $\Omega_{k}\left(H_{n}\right)$. When $k=1$, we have

$$
b_{r_{1}, 1}=w_{r_{1}} .
$$

Under the isomorphism (5), $b_{r_{k}, k}$ modulo $\Omega_{k-1}\left(G_{n}\right)$ corresponds to

$$
(1)_{p^{n-k}, p}^{-1}\left(1+p^{n-k-1}\right)_{p^{n-k}, p}
$$

in $\Omega_{1}\left(G_{n-k+1}\right)$, since in the set Z $/ p^{n-k+1} \mathrm{Z}$ on which $G_{n-k+1}$ acts, $p^{n-k+1} \equiv 0$. Also (17) is $b_{r_{1}, 1}$ in $G_{n-k+1}$; and under the homomorphism (3), $x_{n} \mapsto x_{n-1}$. Therefore defining

$$
b_{i, k}=\left[b_{i+1, k}, x_{n}\right]
$$

for $r_{k}-1 \geqslant i \geqslant 0$, we see from (10) and (11) that

$$
\left\{b_{i, k} \mid r_{k} \geqslant i \geqslant 1\right\} \text { is a basis for } \Omega_{k}\left(H_{n}\right) \text { modulo } \Omega_{k-1}\left(G_{n}\right),
$$

even modulo $\Omega_{k-1}\left(H_{n}\right)$, since (as for $k=1$ ) $b_{i, k} \in H_{n}$ if $k \neq 0$. Also

$$
\left\{b_{i, k} \mid r_{k} \geqslant i \geqslant 0\right\} \text { is a basis for } \Omega_{k}\left(G_{n}\right) \text { modulo } \Omega_{k-1}\left(G_{n}\right) \text {. }
$$

Note that $b_{i, 1}=w_{i}$, for all $i$. Also

$$
\tau_{n} \text { maps } b_{i, 2} \text { in } G_{n} \text { to } b_{i, 1}\left(=w_{i}\right) \text { in } G_{n-1},
$$

for all $i$. We shall use the ' $w$ ' -notation when we wish to emphasise the fact that we are considering $\Omega_{1}\left(G_{n}\right)$ as $X_{n^{-}}$(or $G_{n}$-)module.

By [1], Lemma 3.1 (4),

$$
b_{i, k} \text { commutes with } x_{n, k},
$$

for all $n-1 \geqslant k \geqslant 1$ and $r_{k} \geqslant i \geqslant 0$. Let

$$
B_{k}=\left\langle b_{i, k} \mid r_{k} \geqslant i \geqslant 1\right\rangle,
$$

$1 \leqslant k \leqslant n-1$. Then $B_{k}$ has exponent $p^{k}$ and

$$
\left(\prod_{k=1}^{n-1} B_{k}\right) X_{n}=G_{n}
$$


LEMMA 1. Let $1 \leqslant k \leqslant n-1$. Then

(i) $\left\langle B_{k}, X_{n, k}\right\rangle \cong Z_{p^{k}} \times \cdots \times Z_{p^{k}}$ (with $r_{k}+1$ factors); and

(ii) $\left[w_{i}, B_{k} X_{n, k}\right]=1$, for $p^{n-k}-1 \geqslant i \geqslant 1,2 \leqslant k \leqslant n-1$.

Proof. (i) By (21), $\left[B_{k}, X_{n, k}\right]=1$. Let $C_{k}=C_{G_{n}}\left(x_{n, k}\right)$. So, writing $X=X_{n},\left\langle B_{k}, X\right\rangle \leqslant C_{k}$ and $C_{k}=\left(H_{n} \cap C_{k}\right) X$. Then by (15),

$$
\Omega_{k}\left(C_{k}\right)=\Omega_{k}\left(H_{n} \cap C_{k}\right) X_{n, k}=D_{k},
$$

say. Since $D_{k}$ is a characteristic subgroup of $C_{k}$, it follows that the derived subgroup $D_{k}^{\prime}$ is normalised by $X$. But $D_{k}^{\prime} \leqslant H_{n}$ and so $\left(D_{k}^{\prime}\right)^{G_{n}} \leqslant H_{n}$. Therefore since $H_{n}$ is core-free in $G_{n}$, we must have $D_{k}^{\prime}=1$, i.e.

$$
D_{k} \text { is abelian. }
$$

However, $\left\langle B_{k}, X_{n, k}\right\rangle \leqslant D_{k}$ and so $\left\langle B_{k}, X_{n, k}\right\rangle$ is abelian of exponent $p^{k}$. Thus (i) follows from (19). Observe that

$$
B_{k}=\left\langle b_{r_{k}, k}\right\rangle \times\left\langle b_{r_{k}-1, k}\right\rangle \times \cdots \times\left\langle b_{1, k}\right\rangle,
$$

which is homogeneous of exponent $p^{k}$.

(ii) Let $k \geqslant 2$. By (13),

$$
\left[w_{i}, x_{n, k}\right]=w_{i-p^{n-k}}=1,
$$

for $1 \leqslant i \leqslant p^{n-k}-1$. Therefore $w_{i} \in D_{k}$ and (ii) follows from (23).

We have bases (19) for the $\Omega$-layers of $G_{n}$ (as $X_{n}$-modules) and the homomorphism $\tau_{n}: G_{n} \rightarrow G_{n-1}$ maps these bases to those of $G_{n-1}$ (see (20)). In fact the bases that we have chosen have another very useful property within $G_{n}$ itself. We claim first that

$$
b_{r_{2}, 2}^{p}=b_{r_{2}, 1}\left(=w_{r_{2}}\right) \text {. }
$$

For, from (16) with $k=2$, where the two cycles are disjoint,

$$
b_{r_{2}, 2}^{p}=\prod_{i=0}^{p-1}\left(1+i p^{n-2}\right)_{p^{n-1}, p}^{-1}\left(1+p^{n-3}+i p^{n-2}\right)_{p^{n-1}, p} .
$$

The cycles in (25) are disjoint and hence commute. By (11) and (19) (with $k=2)$,

$$
\Omega_{2}\left(G_{n}\right)=B_{1} B_{2} X_{n, 2}
$$

Therefore by Lemma 1 (i), and using the notation of that Lemma,

$$
D_{2}=\left(D_{2} \cap B_{1}\right) B_{2} X_{n, 2}
$$


and $D_{2}$ is abelian, by (23), and is normalised by $X_{n}$. Thus again by Lemma 1 (i),

$$
D_{2}^{p}=B_{2}^{p} X_{n, 1} \text { is elementary abelian }
$$

of rank $r_{2}+1$ and is an $X_{n}$-submodule of $\Omega_{1}\left(G_{n}\right)$. Hence, setting $W_{j}=\left\langle w_{i} \mid j \geqslant i \geqslant 1\right\rangle$ for $r_{1} \geqslant j \geqslant 0$, we must have

$$
b_{r_{2}, 2}^{p} \in D_{2}^{p} \cap H_{n}=W_{r_{2}} .
$$

Now from the equations following (9) and a simple calculation, we have

$$
b_{r_{2}, 1}=(1)_{p^{n-1}, p}^{-1}(2)_{p^{n-1}, p}^{?} \ldots\left(1+p^{n-3}+(p-1) p^{n-2}\right)_{p^{n-1}, p} .
$$

Observe that the last cycle here is the same as the last cycle in (25). But from (26),

$$
b_{r_{2}, 2}^{p}=\prod_{i=0}^{r_{2}-1} b_{r_{2}-i, 1}^{\alpha_{i}},
$$

for integers $\alpha_{i}$. Therefore, again by the equations following (9), we must have

$$
\alpha_{r_{2}-1}=\alpha_{r_{2}-2}=\cdots=\alpha_{1}=0
$$

and so

$$
b_{r_{2}, 2}^{p} \in\left\langle b_{r_{2}, 1}\right\rangle .
$$

Thus (24) follows by comparing the exponents of (1) ${p^{n-1}, p}$ in (25) and (27).

From (18) we deduce that

$$
b_{r_{2}-1,2}^{p}=\left[b_{r_{2}, 2}, x_{n}\right]^{p}=\left[b_{r_{2}, 2}^{p}, x_{n}\right],
$$

since $B_{2}^{X_{n}} \leqslant D_{2}$, which is abelian. Therefore, by (24) and (9),

$$
b_{r_{2}-1,2}^{p}=\left[b_{r_{2}, 1}, x_{n}\right]=b_{r_{2}-1,1} .
$$

Continuing in this way, we see that

$$
b_{i, 2}^{p}=b_{i, 1}\left(=w_{i}\right),
$$

$r_{2} \geqslant i \geqslant 1$. Also, by (18),

$$
b_{0,2}=\left[b_{1,2}, x_{n}\right] \equiv x_{n, 2} \bmod \Omega_{1}\left(G_{n}\right),
$$

using (12) and (20). Moreover $\left\langle b_{0,2}, x_{n, 2}\right\rangle \leqslant C_{2}$ and

$$
\Omega_{1}\left(G_{n}\right) \cap C_{2}=W_{p^{n-2}-1} X_{n, 1}
$$

(by (13)). Therefore

$$
b_{0,2}^{p}=x_{n, 1}\left(=b_{0,1}\right)
$$


Combining the above results, we can now obtain useful information about adjacent $\Omega$-layers in $G_{n}$. For $2 \leqslant k \leqslant n-1$, we have from (4)

$$
\Omega_{2}\left(G_{n-k+1}\right) \cong \Omega_{2}\left(G_{n} / \Omega_{k-1}\left(G_{n}\right)\right)=\Omega_{k+1}\left(G_{n}\right) / \Omega_{k-1}\left(G_{n}\right) .
$$

Thus we deduce from (28) and (30) that

$$
b_{i, k+1}^{p} \equiv b_{i, k} \bmod \Omega_{k-1}\left(H_{n}\right),
$$

for $r_{k+1} \geqslant i \geqslant 1$; and

$$
b_{0, k+1}^{p} \equiv x_{n, k} \equiv b_{0, k} \bmod \Omega_{k-1}\left(G_{n}\right) .
$$

More information about adjacent $\Omega$-layers of $G_{n}$ will follow from our next key result.

LemMa 2. Let $2 \leqslant k \leqslant n-1$ and for $0 \leqslant i \leqslant p^{k-2}(p-1)$, let

$$
V_{i}=W_{i p^{n-k}-1} \text {. }
$$

Then $B_{k}$ centralises the series

$$
\Omega_{1}\left(H_{n}\right)=W_{r}=V_{p^{k-2}(p-1)}>\cdots>V_{i}>V_{i-1}>\cdots>V_{0}=1
$$

of length $p^{k-2}(p-1)$.

Proof. By Lemma 1 (ii), $B_{k}$ centralises $V_{1}$. We argue by induction on $i$ increasing and suppose that $\left[V_{i+1}, B_{k}\right] \leqslant V_{i}$ for some $i \geqslant 0$. Then by (13)

$$
\left[V_{i+2}, X_{n, k}\right]=V_{i+1} X_{n, 1},
$$

and so

$$
\left[V_{i+2}, X_{n, k}, B_{k}\right] \leqslant V_{i} .
$$

Since $\left[X_{n, k}, B_{k}\right]=1$, by Lemma 1 (i), the Three Subgroup Lemma (see for example [8], Lemma 2.13) implies

$$
\left[B_{k}, V_{i+2}, X_{n, k}\right] \leqslant V_{i} X_{n, 1}\left(\triangleleft G_{n}\right) .
$$

Therefore $\left[B_{k}, V_{i+2}\right] \leqslant V_{i+1} X_{n, 1}$, again by (13). Thus

$$
\left[B_{k}, V_{i+2}\right] \leqslant V_{i+1} X_{n, 1} \cap H_{n}=V_{i+1}
$$

and the Lemma follows.

CoRollary 1. For $0 \leqslant i \leqslant n-2$, the factor group $\Omega_{i+2}\left(G_{n}\right) / \Omega_{i}\left(G_{n}\right)$ is nilpotent of class $p-1$ and hence regular. 
Proof. By (31) we may assume that $i=0$. Take $k=2$ in Lemma 2 . Then $B_{2}$ centralises the series (33) of length $p-1$. Also, modulo $X_{n, 1}, X_{n, 2}$ centralises this series (by (13)). Therefore, since $B_{2} X_{n, 2}$ is abelian (by Lemma 1 (i)), the subgroup

$$
\Omega_{2}\left(G_{n}\right)=B_{1} B_{2} X_{n, 2}
$$

is nilpotent of class at most $p-1$. In fact the class is exactly $p-1$, because of the action of $X_{n, 2}$ on $\Omega_{1}\left(G_{n}\right)=W_{r} X_{n, 1}$. The regularity follows, for example, from [3], Corollary 12.3.1.

\section{Subgroups of $G_{n}$.}

We can now describe all the quasinormal subgroups of $G_{n}$ lying in $H_{n}$ and of exponent $p$.

THEOREM 2. Let $n \geqslant 2$. Then a quasinormal subgroup of $G_{n}$ of exponent $p$, lying in $H_{n}$, has the form $W_{i}$, for some $i$. Moreover $W_{i}$ qn $G_{n}$ if and only if $1 \leqslant i \leqslant p^{2}-1$.

Proof. Observe from (6) that when $n=2, i \leqslant p-2$; and when $n=3$, $i \leqslant p(p-1)-1$. Therefore in both these cases we are saying that $W_{i} q n G=G_{n}$ for all $i$ for which $W_{i}$ is defined.

Let $Q q n G$ with $Q$ of exponent $p$ and lying in $H=H_{n}$. Then $Q \leqslant W_{r_{1}}=\Omega_{1}(H)$. Also $Q X_{n}$ is a subgroup and $\Omega_{1}\left(Q X_{n}\right)=Q X_{n, 1}$. Thus $Q X_{n, 1}$ is an $X_{n}$-submodule of $\Omega_{1}(G)$ and so, since indecomposable modules are uniserial (see [4], Theorem VII, 5.3), $Q X_{n, 1}=W_{i} X_{n, 1}$, for some $i$. Hence $Q=W_{i}$.

We establish first the sufficiency of the condition on $i$. Therefore suppose that $i \leqslant p^{2}-1$ and let $g=h y$, where $h \in H$ and $y \in X=X_{n}$, and put $K=\langle g\rangle$. We show that $W_{i} K$ is a subgroup by considering 3 possibilities.

(i) Suppose that $y \in X^{p^{2}}=X_{n, n-2}$. Then $y$ centralises $W_{i}$, by (13). Since $W_{i} \triangleleft H$, it follows that $g$ normalises $Q$.

(ii) Suppose that $\langle y\rangle=X$. Then by [1], Lemma 3.12 (ii), $\Omega_{1}(K)=X_{n, 1}$. Therefore $W_{i} K=W_{i} X_{n, 1} K$ is a subgroup, since $W_{i} X_{n, 1} \triangleleft G$.

(iii) Finally suppose that $\langle y\rangle=X^{p}$. Clearly we may assume that $n \geqslant 3$. By Corollary $1, \Omega_{n-1}(G) / \Omega_{n-3}(G)$ is regular with elementary abelian derived subgroup. Therefore

$$
g^{p} \equiv h^{p} y^{p} \bmod \Omega_{n-3}(G) .
$$


Also

$$
h^{p} \in\left\langle b_{j, n-2} \mid 1 \leqslant j \leqslant p-2\right\rangle \bmod \Omega_{n-3}(H),
$$

by (32) and (14). Continuing taking $p$-th powers in this way, we obtain

$$
g^{p^{n-2}} \in W_{p-2} X_{n, 1}
$$

Moreover $|H K: H|=|H\langle y\rangle: H|=|\langle y\rangle|=p^{n-1}$. Therefore $g^{p^{n-2}} \notin H$.

Now consider $W_{i} K$. If $i \leqslant p-1$, then $y$ centralises $W_{i}$ (by (13)) and so again $g$ normalises $W_{i}$, i.e. $W_{i} K$ is a subgroup. On the other hand if $p \leqslant i\left(\leqslant p^{2}-1\right)$, then by $(34)$

$$
W_{i} K=W_{i} X_{n, 1} K
$$

and this is a subgroup as in (ii).

Conversely, suppose, for a contradiction, that $W_{i} q n G$ where $i \geqslant p^{2}$. Then by (6), $n \geqslant 4$. Let $g=b_{1, n-1} x_{n, n-2}$ and $K=\langle g\rangle$. We have $\left[b_{1, n-1}, x_{n, n-1}\right]=1$, by (21). So

$$
g^{p^{n-2}}=b_{1, n-1}^{p^{n-2}}=b_{1,1}=w_{1},
$$

using the argument of (iii) above. Consider the subgroup $W_{i} K$. Since $\Omega_{1}(G)$ is elementary abelian, an element of order $p$ in $W_{i} K$ must have the form $w g_{1}$, where $w \in W_{i}$ and $g_{1} \in \Omega_{1}(K)=W_{1}$ (by (35). Thus $\Omega_{1}\left(W_{i} K\right)=$ $W_{i} \triangleleft W_{i} K$. Since $W_{i} \triangleleft H$, it follows that $x_{n, n-2}$ also normalises $W_{i}$. But $\left[w_{p^{2}}, x_{n, n-2}\right]=x_{n, 1}$ (by (13)), a contradiction. This completes the proof of Theorem 2.

For the next theorems we need to be able to identify certain subgroups of $G_{n}$. Let $n \geqslant 2, n-1 \geqslant k \geqslant 1$ and $r_{k} \geqslant \ell_{k} \geqslant 1$. Define

$$
B_{\ell_{k}, k}=\left\langle b_{j, k} \mid 1 \leqslant j \leqslant \ell_{k}\right\rangle,
$$

which is isomorphic to $Z_{p^{k}} \times \cdots \times Z_{p^{k}}$ ( $\ell_{k}$ copies), by Lemma 1 (i).

Note that $B_{\ell_{1}, 1}=W_{\ell_{1}}$.

LEMMA 3. Let $n \geqslant 2, \quad r_{k} \geqslant \ell_{k} \geqslant p^{n-k-1}-1$ for $1 \leqslant k \leqslant n-3, \quad r_{n-2} \geqslant$ $\ell_{n-2} \geqslant \ell_{n-1}$ and $r_{n-1} \geqslant \ell_{n-1} \geqslant 1$. Then

$$
L=B_{\ell_{1}, 1} B_{\ell_{2}, 2} \ldots B_{\ell_{n-1}, n-1} X_{n, n-1}
$$

is a subgroup of $G_{n}$; and

$$
B_{\ell_{1}, 1} B_{\ell_{2}, 2} \ldots B_{\ell_{n-1}, n-1}=L \cap H_{n}
$$

is a subgroup of $H_{n}$. 
PRoof. If $n=2$, then $L=W_{\ell_{1}} X_{n, 1} \triangleleft G_{2}$. If $n=3$, then $L=W_{\ell_{1}} B_{\ell_{2}, 2} X_{n, 2}$ with $r_{1}=p(p-1)-1 \geqslant \ell_{1} \geqslant \ell_{2}$ and $r_{2}=p-2 \geqslant \ell_{2} \geqslant 1$. By Lemma 1 (i), $B_{\ell_{2}, 2} X_{n, 2}$ is a subgroup. Also $W_{\ell_{1}} X_{n, 1} \triangleleft G_{3}$. Since $L$ is the product of these two subgroups, again $L$ is a subgroup.

Now suppose that the Lemma is true for $n-1$, some $n \geqslant 4$, and argue by induction on $n$. Then from (4) we obtain

$$
L \Omega_{1}\left(G_{n}\right)=W_{r_{1}} L
$$

is a subgroup. Recall that $C_{2}=C_{G_{n}}\left(x_{n, 2}\right)$. It follows from (13), (22) and Lemma 1 (i), that

$$
C_{2}=W_{p^{n-2}-1} B_{r_{2}, 2} \ldots B_{r_{n-1}, n-1} X_{n}
$$

Then

$$
\begin{gathered}
W_{r_{1}} L \cap C_{2}=W_{r_{1}} B_{\ell_{2}, 2} \ldots B_{\ell_{n-1}, n-1} X_{n, n-1} \cap C_{2} \\
=W_{p^{n-2}-1} B_{\ell_{2}, 2} \ldots B_{\ell_{n-1}, n-1} X_{n, n-1}
\end{gathered}
$$

is a subgroup. Since $\ell_{1} \geqslant p^{n-2}-1$, forming the product of this subgroup with the normal subgroup $W_{\ell_{1}} X_{n, 1}$, we obtain $L$. So $L$ is a subgroup.

Equation (37) follows from Dedekind's Intersection Lemma.

The quasinormal subgroups of exponent $p^{n-1}$ that we shall exhibit in Theorem 3 all have the form $L \cap H_{n}$ given by (37); and those of exponent $p^{n-2}$ in Theorem 4 have the form $\Omega_{n-2}\left(L \cap H_{n}\right)$. As a consequence of the next result,we see that these subgroups are actually normal in $H_{n}$.

LEMMA 4. Let $n \geqslant 3, r_{k} \geqslant \ell_{k} \geqslant r_{k}-p^{2}$ for $1 \leqslant k \leqslant n-3, r_{n-2} \geqslant \ell_{n-2} \geqslant \ell_{n-1}$ and $r_{n-1} \geqslant \ell_{n-1} \geqslant 1$. Define $L$ as in (36). Then

(i) $L$ is a subgroup of $G_{n}$; and

(ii) for $1 \leqslant k \leqslant n-1$,

$$
\Omega_{k}(L)=B_{\ell_{1}, 1} B_{\ell_{2}, 2} \ldots B_{\ell_{k}, k} X_{n, k}=L_{k},
$$

say; and $L_{k} \triangleleft G_{n}$ for $1 \leqslant k \leqslant n-2$.

Moreover, if $r_{k} \geqslant \ell_{k} \geqslant r_{k}-p$ for $1 \leqslant k \leqslant n-2$ and $r_{n-1} \geqslant \ell_{n-1} \geqslant 1$, then

(iii) again $L$ is a subgroup of $G_{n}$ and $L \triangleleft G_{n}$.

Proof. (i) The hypotheses here imply those of Lemma 3. For, with $n \geqslant 3$ and $1 \leqslant k \leqslant n-3$,

$$
\begin{gathered}
r_{k}-p^{2}-\left(p^{n-k-1}-1\right)=p^{n-k-1}(p-1)-p^{2}-p^{n-k-1}=p^{n-k-1}(p-2)-p^{2} \\
\geqslant p^{2}(p-2)-p^{2} \geqslant 0 .
\end{gathered}
$$

Therefore $L$ is a subgroup of $G_{n}$. 
(ii) It is easy to check that, for $1 \leqslant k \leqslant n-2, \ell_{k} \geqslant \ell_{k+1}$. We claim that, for $2 \leqslant k \leqslant n-1$,

$$
\left(B_{\ell_{k}, k} X_{n, k}\right)^{p} \subset B_{\ell_{1}, 1} B_{\ell_{2}, 2} \ldots B_{\ell_{k-1}, k-1} X_{n, k-1} .
$$

We do not know yet that the right side of (39) is a subgroup. In order to prove (39), suppose that $k=2$. Then $B_{\ell_{k}, k} X_{n, k} \cong Z_{p^{2}} \times \cdot \cdot \times Z_{p^{2}}\left(\ell_{k}+1\right.$ copies), by Lemma 1 (i). Thus (39) follows from (28), since $\ell_{1} \geqslant \ell_{2}$. Therefore (39) is true for $n=3$. We suppose that (39) is true for $G_{n-1}(n \geqslant 4)$ and $2 \leqslant k \leqslant n-2$ and argue by induction on $n$. Then for $3 \leqslant k \leqslant n-1$, we have from (20)

$$
\left(B_{\ell_{k}, k} X_{n, k}\right)^{p} \subset W_{r_{1}} B_{\ell_{2}, 2} \ldots B_{\ell_{k-1}, k-1} X_{n, k-1} .
$$

However, the left side of (40) and all but the first factor on the right belong to $C_{2}\left(=C_{G_{n}}\left(x_{n, 2}\right)\right)$. Thus intersecting (40) with $C_{2}$ gives

$$
\begin{aligned}
\left(B_{\ell_{k}, k} X_{n, k}\right)^{p} & \subset W_{p^{n-2}-1,1} B_{\ell_{2}, 2} \ldots B_{\ell_{k-1}, k-1} X_{n, k-1} \\
& \subset B_{\ell_{1}, 1} B_{\ell_{2}, 2} \ldots B_{\ell_{k-1}, k-1} X_{n, k-1},
\end{aligned}
$$

since one checks easily that

$$
\ell_{1} \geqslant p^{n-2}-1
$$

Therefore (39) is true.

Now we can prove (38). Clearly $\Omega_{n-1}(L)=L=L_{n-1}$. We proceed by induction on $k$ decreasing and suppose that (38) is true for some $k \leqslant n-1$. Certainly

$$
\Omega_{k-1}(L) \supset B_{\ell_{1}, 1} \ldots B_{\ell_{k-1}, k-1} X_{n, k-1} .
$$

If the inclusion (42) is strict, then there is an element $g \in B_{\ell_{k}, k} X_{n, k}$ with $|g|=p^{k-1}$ and $g \notin L_{k-1}$. But by Lemma 1 (i), $g \in\left(B_{\ell_{k}, k} X_{n, k}\right)^{p}$, contradicting (39). Therefore (42) is an equality. Thus our induction argument goes through and we have proved (38).

The second part of (ii) will follow from

$$
L_{n-2} \triangleleft G_{n} \text {. }
$$

When $n=3, L=W_{\ell_{1}} B_{\ell_{2}, 2} X_{3,2}$ and $L_{1}=W_{\ell_{1}} X_{3,1} \triangleleft G_{3}$. Therefore we suppose that $n \geqslant 4$ and that (43) is true for $G_{n-1}$ and proceed by induction. Again from (20)

$$
W_{r_{1}} B_{\ell_{2}, 2} \ldots B_{\ell_{n-2}, n-2} X_{n, n-2} \triangleleft G_{n} ;
$$

and intersecting with $C_{2}$ gives

$$
W_{p^{n-2}-1} B_{\ell_{2}, 2} \ldots B_{\ell_{n-2}, n-2} X_{n, n-2} \triangleleft C_{2} .
$$


Thus from (41) and $W_{\ell_{1}} X_{n, 1} \triangleleft G_{n}$, we see that $L_{n-2}$ is normalised by $C_{2}$. However, $\left[W_{r_{1}}, L_{n-2}\right] \leqslant W_{r_{1}-p^{2}} X_{n, 1}$, by Lemma 2 , and so $W_{r_{1}}$ normalises $L_{n-2}$. Since $W_{r_{1}} C_{2}=G_{n}$, (43) follows.

(iii) It is easy to check that the hypotheses here imply those of parts (i) and (ii). So $L$ is a subgroup. Now however

$$
\left[W_{r_{1}}, L\right] \leqslant W_{r_{1}-p} X_{n, 1} \leqslant L,
$$

by Lemma 2 , and so $W_{r_{1}}$ normalises $L$. Also when $n=3$, by considering $L$ modulo $\Omega_{1}\left(G_{3}\right)$ and intersecting with $C_{2}$, we see that $L \triangleleft G_{n}$. Then the induction argument used to prove (43) can be applied here to give $L \triangleleft G_{n}$ for all $n$.

\section{Quasinormal subgroups of large exponent.}

We shall see that the quasinormal subgroups $Q$ of $G_{n}$ lying in $H_{n}$, other than those of Theorem 2, all have exponent $p^{m}$ for $m=n-2$ or $n-1$. Also $\Omega_{k}(Q)$ modulo $\Omega_{k-1}\left(H_{n}\right)$ has 'large' rank, for all $1 \leqslant k \leqslant m-1$. We begin with those of exponent $p^{n-1}$.

THEOREM 3. (i) Let $n \geqslant 3$ and $Q$ be a quasinormal subgroup of $G_{n}$ of exponent $p^{n-1}$ and lying in $H_{n}$. Then for $1 \leqslant k \leqslant n-1$,

$$
\Omega_{k}(Q) \equiv B_{\ell_{k}, k} \bmod \Omega_{k-1}\left(H_{n}\right) ;
$$

and $\ell_{k} \geqslant r_{k}-p$, for $1 \leqslant k \leqslant n-2$, and $\ell_{n-1} \geqslant 1$.

(ii) Again let $n \geqslant 3$ and

$$
Q=\prod_{k=1}^{n-1} B_{\ell_{k}, k}
$$

with $\ell_{k} \geqslant r_{k}-p$ for $1 \leqslant k \leqslant n-2$ and $\ell_{n-1} \geqslant 1$. Then $Q$ is a subgroup of $H_{n}$ of exponent $p^{n-1}$. Moreover $Q q n G_{n}$ if and only if

$$
\ell_{k} \geqslant r_{k}-p+1 \text {, }
$$

for $1 \leqslant k \leqslant n-2$.

Proof. (i) Since $Q X_{n}$ is a subgroup, we see that $\Omega_{1}\left(Q X_{n}\right)$ is an $X_{n}$ module contained in $\Omega_{1}\left(G_{n}\right)$ and therefore has the form $W_{i} X_{n, 1}$. Also there is an element $h b_{1, n-1} \in Q$ with $h \in \Omega_{n-2}\left(H_{n}\right)$, by Theorem 2 . Thus since $W_{r_{1}}$ must normalise $Q$,

$$
\left[W_{r_{1}}, h b_{1, n-1}\right] \leqslant Q \cap W_{r_{1}-p},
$$


by Lemma 2. However

this commutator subgroup does not belong to $W_{r_{1}-p-1}$.

For, otherwise we would have $\left[W_{r_{1}}, b_{1, n-1}\right] \leqslant W_{r_{1}-p-1}$. Then conjugating by $x_{n}$, we would obtain (using (18))

$$
\left[W_{r_{1}}, b_{1, n-1} b_{0, n-1}\right] \leqslant W_{r_{1}-p-1} X_{n, 1} .
$$

But $b_{0, n-1} \equiv x_{n, n-1} \bmod \Omega_{n-2}\left(G_{n}\right)$, using (29) and the homomorphism $\tau_{n}$. Thus $x_{n, n-1}$ would centralise $W_{r_{1}} X_{n, 1} / W_{r_{1}-p-1} X_{n, 1}$, a contradiction.

Thus (44) is true for $k=1$. The rest of (i) follows by induction on $n$ from $G_{n} / \Omega_{1}\left(G_{n}\right) \cong G_{n-1}$.

(ii) The hypotheses here imply those of Lemma 4. Thus $Q$ is a subgroup of $H_{n}$ of exponent $p^{n-1}$ (of the form (37)).

Now suppose that $Q$ qn $G_{n}$. Assume for the moment that

$$
\ell_{1} \geqslant r_{1}-p+1
$$

Then we obtain (45), again by induction on $n$. So it suffices to prove (47).

Let $g=w_{r_{1}} x_{n}$ and $K=\langle g\rangle$. Then

$$
\Omega_{1}(K)=\left\langle x_{n, 1}\right\rangle
$$

by [1], Lemma 3.12 (2). In fact this follows easily from a simple module calculation. Thus using additive notation,

$$
\begin{aligned}
g^{p} & =\left(w_{r_{1}} x_{n}\right)^{p}=w_{r_{1}}\left(1+x_{n}^{-1}+x_{n}^{-2}+\cdots+x_{n}^{-(p-1)}\right) x_{n, n-1} \\
& =w_{r_{1}}\left(x_{n}^{-1}-1\right)^{p-1} x_{n, n-1} \equiv w_{r_{1}}\left(x_{n}-1\right)^{p-1} x_{n, n-1} \bmod W_{r_{1}-p} X_{n, 1} \\
& \equiv w_{r_{1}-p+1} x_{n, n-1} \bmod W_{r_{1}-p} X_{n, 1} .
\end{aligned}
$$

(The appearance of $w_{r_{1}-p+1}$ here is the clue to establishing (47).) Similarly

$$
g^{p^{2}} \equiv w_{r_{1}-p^{2}+1} x_{n, n-2} \bmod W_{r_{1}-p^{2}} X_{n, 1}
$$

(We can obtain (48) by computing $g^{p^{n-1}}$ in this way.) Since $\ell_{1}>r_{1}-p^{2}+1$, it follows from (48) that

$$
x_{n, n-2} \in Q K
$$

Also $b_{1, n-1} \in Q$, by hypothesis, and so $Q K$ contains

$$
\left[b_{1, n-1}, g\right]=\left[b_{1, n-1}, x_{n}\right]\left[b_{1, n-1}, w_{r_{1}}\right]^{x_{n}} .
$$

However, by Lemma 2,

$$
\left[b_{1, n-1}, w_{r_{1}}\right]^{x_{n}} \in W_{\ell_{1}}^{x_{n}}=W_{\ell_{1}}^{g} \leqslant Q K .
$$




\section{Hence}

$$
Q K \text { contains }\left[b_{1, n-1}, x_{n}\right]=g_{1},
$$

say. Now $g_{1} \in Q^{G_{n}} \leqslant Q X_{n, n-1}$, since $Q X_{n, n-1} \triangleleft G_{n}$, by Lemma 4 (iii). From (4) and (12) we have

$$
g_{1}=x_{n, n-1} g_{2}
$$

where $g_{2} \in \Omega_{n-2}\left(G_{n}\right)=\Omega_{n-2}\left(H_{n}\right) X_{n, n-2}$. Therefore $g_{2}=h y$, with $h \in$ $\Omega_{n-2}\left(H_{n}\right)$ and $y \in X_{n, n-2}$. Thus $y \in Q K$, by (50). Then

$$
\begin{gathered}
g_{1} \in Q X_{n, n-1} \Rightarrow g_{2} \in Q X_{n, n-1} \Rightarrow h \in Q X_{n, n-1} \cap H_{n}=Q \Rightarrow g_{2} \in Q K \Rightarrow \\
\Rightarrow x_{n, n-1} \in Q K,
\end{gathered}
$$

by (51).

Finally from (49) we now obtain

$$
w_{r_{1}-p+1} \in Q K \cap H_{n}=Q .
$$

Thus $\ell_{1} \geqslant r_{1}-p+1$, as required.

Conversely, suppose that $\ell_{k} \geqslant r_{k}-p+1$ for $1 \leqslant k \leqslant n-2$ and $\ell_{n-1} \geqslant 1$. We show that $Q q n G_{n}$. Let $g=h y$, where $h \in H_{n}, y \in X_{n}$, and let $K=\langle g\rangle$. It suffices to show that $Q K$ is a subgroup. Let $y \in X_{n, k} \backslash X_{n, k-1}$, where $0 \leqslant k \leqslant n$. (Assume that $X_{n,-1}=1$.) We claim that

$$
g^{p} \in Q X_{n, k-1} .
$$

Note that $Q X_{n, n-1} \triangleleft G_{n}$, by Lemma 4 (iii). Thus $Q X_{n, i}$ is a subgroup for all $i$; and

$$
Q \triangleleft H_{n} .
$$

Let $h=w b_{2} b_{3} \ldots b_{n-1}$, where $w \in W_{r_{1}}$ and $b_{i} \in B_{i}, 2 \leqslant i \leqslant n-1$. Considering $\Omega_{1}\left(G_{n}\right)$ as $G_{n}$-module, we have

$$
g^{p}=\left(w b_{2} \ldots b_{n-1} y\right)^{p}=w\left(\left(b_{2} \ldots b_{n-1} y\right)^{-1}-1\right)^{p-1}\left(b_{2} \ldots b_{n-1} y\right)^{p} .
$$

Thus

$$
g^{p} \in W_{r_{1}-p+1} C_{2},
$$

by Lemma 1 . We prove (52) by induction on $n$.

Suppose $n=3$. If $k \leqslant 2$, then $g^{p} \in W_{p-2} X_{3, k-1}$, by Corollary 1 , and so $g^{p} \in Q X_{3, k-1}$. If $k=3$, then by (54)

$$
g^{p} \in W_{r_{1}} X_{3,2} \cap W_{r_{1}-p+1} C_{2}=\left(W_{r_{1}} \cap W_{r_{1}-p+1} C_{2}\right) X_{3,2} \leqslant Q X_{3,2} .
$$


So (52) holds for $n=3$. We proceed by induction on $n$, assuming that $n \geqslant 4$ and that (52) holds in $G_{n-1}$. Thus by induction (using $G_{n} / \Omega_{1}\left(G_{n}\right) \cong G_{n-1}$ ) and (54), we have

$$
\begin{gathered}
g^{p} \in W_{r_{1}} X_{n, 1} Q X_{n, k-1} \cap W_{r_{1}-p+1} C_{2} \leqslant\left(W_{r_{1}} Q \cap W_{r_{1}-p+1} C_{2}\right) X_{n, 1} X_{n, k-1} \\
=W_{r_{1}-p+1}\left(W_{r_{1}} Q \cap C_{2}\right) X_{n, 1} X_{n, k-1} \leqslant W_{r_{1}-p+1}\left(W_{p^{n-2}-1} Q\right) X_{n, 1} X_{n, k-1},
\end{gathered}
$$

by the argument used in Lemma 3. Therefore $g^{p} \in Q X_{n, 1} X_{n, k-1}$. So (52) follows if $k \geqslant 2$. But if $k \leqslant 1$, then $g^{p} \in H_{n}$ and so $g^{p} \in Q\left(=Q X_{n, k-1}\right)$. Thus we have established (52).

Now from (52) we obtain $Q\left\langle g^{p}\right\rangle \leqslant Q X_{n, k-1}$. But

$$
\left|Q\left\langle g^{p}\right\rangle\right|=\left|Q \|\left\langle g^{p}\right\rangle: Q \cap\left\langle g^{p}\right\rangle\right|=|Q| p^{k-1}=\left|Q X_{n, k-1}\right| .
$$

Therefore

$$
Q\left\langle g^{p}\right\rangle=Q X_{n, k-1}
$$

a subgroup. By Lemma 4

$$
\Omega_{k-1}\left(Q X_{n, n-1}\right)=\Omega_{k-1}(Q) X_{n, k-1}=N,
$$

say, and $N \triangleleft G_{n}$. If $k \leqslant n-1$, then by Lemma 1 (i), $X_{n, k}$ centralises $Q$ modulo $N$ and therefore $g$ normalises $Q$ (by (53)). Otherwise $k=n$ and then $N=Q X_{n, n-1}=Q\left\langle g^{p}\right\rangle$, by (55); i.e. $Q K$ is a subgroup.

This completes the proof of Theorem 3.

Next we deal with the quasinormal subgroups of exponent $p^{n-2}$. The argument follows that of Theorem 3, though there added complications. Establishing the necessity of our conditions for quasinormality involves considering the product $Q K$ for $K=\left\langle w_{r_{1}} b_{1, n-1} x_{n, 2}\right\rangle$ rather than $\left\langle w_{r_{1}} x_{n}\right\rangle$; and the prime 3 causes problems.

THEOREM 4. (i) Let $n \geqslant 4$ and $Q q n G_{n}, Q \leqslant H_{n}$ and $Q$ have exponent $p^{n-2}$. Then for $1 \leqslant k \leqslant n-2$,

$$
\Omega_{k}(Q) \equiv B_{\ell_{k}, k} \bmod \Omega_{k-1}\left(H_{n}\right) ;
$$

and $\ell_{k} \geqslant r_{k}-p^{2}$, for $1 \leqslant k \leqslant n-3$, and $\ell_{n-2} \geqslant 1$.

(ii) Again let $n \geqslant 4$ and

$$
Q=\prod_{k=1}^{n-2} B_{\ell_{k}, k}
$$

with $\ell_{k} \geqslant r_{k}-p^{2}$ for $1 \leqslant k \leqslant n-3$ and $\ell_{n-2} \geqslant 1$. Then $Q$ is a subgroup of $H_{n}$ 
of exponent $p^{n-2}$. Moreover $Q$ qn $G_{n}$ if and only if

$$
\ell_{k} \geqslant r_{k}-p(p-1)
$$

for $1 \leqslant k \leqslant n-3$ and $\ell_{n-2} \geqslant p-2$.

Proof. (i) We argue exactly as in Theorem 3 (i) to obtain the form (56). Then for the restrictions on $\ell_{k}$ we use the fact that there is an element $h b_{1, n-2} \in Q$, with $h \in \Omega_{n-3}\left(H_{n}\right)$. Thus $\left[W_{r_{1}}, h b_{1, n-2}\right]$ lies in $Q \cap W_{r_{1}-p^{2}}$ (by Lemma 2), but not in $W_{r_{1}-p^{2}-1}$.

(ii) With $L$ defined as in Lemma 4, we have

$$
Q=\Omega_{n-2}(L) \cap H_{n} .
$$

Hence $Q$ is a subgroup.

Now suppose that $Q$ qn $G_{n}$. We shall show that

$$
\ell_{1} \geqslant r_{1}-p(p-1)
$$

and

$$
\ell_{2} \geqslant p-2 \text { when } n=4 .
$$

Then the necessity of our conditions will follow by induction on $n$, as in Theorem 3. We shall prove that

$$
w_{p^{n-2}} \in Q,
$$

i.e. $\ell_{1} \geqslant p^{n-2}$. Assume this for the moment and let

$$
g=w_{r_{1}} b_{1, n-1} x_{n, 2}, \quad K=\langle g\rangle .
$$

Then since $Q K$ is a subgroup, (59) implies

$$
\left[w_{p^{n-2}}, g\right] \in Q K .
$$

Therefore substituting for $g$ and expanding (using (13)), we get

$$
\left[w_{p^{n-2}}, b_{1, n-1}\right] x_{n, 1} \in Q K .
$$

Thus $x_{n, 1} \in Q K$. Considering actions on $\Omega_{1}\left(G_{n}\right)$ and using Lemma 2, we obtain

$$
g^{p}=w b_{1, n-1}^{p} x_{n, 1}^{\varepsilon},
$$

where

$$
w \in W_{r_{1}-p(p-1)} \backslash W_{r_{1}-p(p-1)-1} .
$$

The appearance of the suffix $r_{1}-p(p-1)$ in (60) is the clue to establishing 
(57). Recall that $b_{1, n-1}$ and $x_{n, 2}$ commute, by Lemma 1 (i). Also $\varepsilon$ is not divisible by $p$, since $g^{p} \notin H_{n}$. By (39), $b_{1, n-1}^{p} \in Q$. Therefore $w x_{n, 1}^{\varepsilon} \in Q K$ and so $w \in Q K$. But if (57) is false, then $w \notin Q$. Thus $\langle w\rangle \times\left\langle x_{n, 1}\right\rangle$ is disjoint from $Q$ and embeds in the chain $[Q K / K]$, a contradiction. Then (57) will follow from (59).

We have

$$
\ell_{1} \geqslant r_{1}-p^{2}
$$

from (i), i.e.

$$
\ell_{1} \geqslant p^{n-2}(p-2)-1 .
$$

Thus if $p \geqslant 5$, then $\ell_{1} \geqslant p^{n-2}$ and we have (59). Therefore we may suppose that $p=3$.

Let $g=w_{r_{1}} x_{n}$ and $K=\langle g\rangle$. Then $Q K$ is a subgroup and we have

$$
\begin{aligned}
g^{p^{n-2}} & =w_{r_{1}}\left(x_{n}^{-1}-1\right)^{p^{n-2}-1} x_{n, 2} \\
& \in Q K \cap\left(W_{r_{1}-p^{n-2}+1} X_{n, 2} \backslash W_{r_{1}-p^{n-2}} X_{n, 2}\right) .
\end{aligned}
$$

Also $b_{1,2} \in Q$ (by definition) and thus $Q K$ contains

$$
\left[b_{1,2}, w_{r_{1}} x_{n}\right]=\left[b_{1,2}, x_{n}\right]\left[b_{1,2}, w_{r_{1}}\right]^{x_{n}} .
$$

Here the first factor on the right has the form $w x_{n, 2}$, where

$$
w \in W_{p^{n-2}-1} \leqslant Q,
$$

by (29), the fact that $C_{2}$ contains $b_{1,2}$ and $x_{n}$, and (62); and by Lemma 2 the second factor lies in $W_{r_{1}-p^{n-2}} X_{n, 1} \leqslant Q K$, by (61) and the fact that

$$
\Omega_{1}(K)=X_{n, 1}
$$

(see [1], Lemma 3.12 (2)). Therefore $x_{n, 2} \in Q K$ and hence, by (63),

$$
W_{r_{1}-p^{n-2}+1} \leqslant Q K \cap H_{n}=Q
$$

(again using (64)). Thus $\ell_{1} \geqslant r_{1}-p^{n-2}+1=p^{n-2}(p-2)$ and (59) follows.

It remains to prove (58). Here $n=4$. Let $g=b_{p-2,3} x_{4,2}$. Then

$$
g^{p}=w b_{p-2,2} x_{4,1}
$$

where $w \in W_{p^{2}-1}$ (using (28) and the facts that $b_{p-2,3}$ and $x_{4,2}$ commute and $\left.g \in C_{2}\right)$. Thus

$$
g^{p^{2}}=w_{p-2}
$$


Let $K=\langle g\rangle$ and consider the subgroup $Q K$. If $u v$ has order $p$ with $u \in Q$ and $v \in K$, then assuming (58) is false, we must have

$$
v \in\left\langle g^{p^{2}}\right\rangle .
$$

But by (57), $\ell_{1} \geqslant r_{1}-p(p-1)=p(p-1)^{2}-1>p-2$ and hence by (65), $v \in Q$. Therefore $u v \in \Omega_{1}(Q)=W_{\ell_{1}}=\Omega_{1}(Q K) \triangleleft Q K$ and so $g$ normalises $W_{\ell_{1}}$. Thus $x_{4,2}$ normalises $W_{\ell_{1}}$. However, $W_{p^{2}} \leqslant W_{\ell_{1}}$ (by (59)), contradicting $\left[W_{p^{2}}, x_{4,2}\right]=X_{4,1}$. This proves (58) and we have established the necessary conditions in (ii).

Conversely suppose that

$$
\ell_{k} \geqslant r_{k}-p(p-1), \text { for } 1 \leqslant k \leqslant n-3, \text { and } \ell_{n-2} \geqslant p-2 .
$$

Let $g=h y, h \in H_{n}, y \in X_{n}$, and $K=\langle g\rangle$. We show that

$$
Q K \text { is a subgroup. }
$$

By Lemma 4 (ii),

$$
Q X_{n, n-2} \triangleleft G_{n}
$$

and so $Q \triangleleft H_{n}$. Thus we may suppose that $y \notin X_{n, 1}$. Suppose that $y \in X_{n, k} \backslash X_{n, k-1}, 2 \leqslant k \leqslant n-1$. It follows from (67) that $Q X_{n, k-1}$ is a subgroup. Then by analogy with (52) in the proof of Theorem 3 (ii), we have

$$
g^{p} \in Q X_{n, k-1} .
$$

For, the argument used to prove (54) gives $g^{p} \in W_{r_{1}-p(p-1)} C_{2}$. Then (68) follows by induction on $n$, as in Theorem 3 (ii). Analogous to (55) we obtain

$$
Q\left\langle g^{p}\right\rangle=Q X_{n, k-1}
$$

and in the same way we deduce that $Q K$ is a subgroup.

Finally suppose that $K=X_{n}$. Then $g^{p} \in H_{n} X_{n, n-1}$ and, by the above, $Q\left\langle g^{p^{2}}\right\rangle=Q X_{n, n-2} \triangleleft G_{n}$. Thus (66) follows and $Q q n G_{n}$.

Our final result is surely the most striking, showing that a finite $p$ group can be remarkably devoid of quasinormal subgroups throughout most of its structure.

THEOREM 5. Let $n \geqslant 5$. Then there are no quasinormal subgroups of $G_{n}$, lying in $H_{n}$ and of exponent $p^{k}$, for $2 \leqslant k \leqslant n-3$.

PRooF. Using $G_{n} / \Omega_{1}\left(G_{n}\right) \cong G_{n-1}$ and induction, it suffices to prove that there are no quasinormal subgroups of exponent $p^{2}$. Thus suppose that 
$Q q n G_{n}, Q \leqslant H_{n}$ and $Q$ has exponent $p^{2}$. As we saw in Theorems 3 (i) and 4 (i), we must have

$$
\Omega_{1}(Q)=W_{i} \text { and } Q \equiv B_{\ell_{2}, 2} \bmod W_{r_{1}}
$$

for some $i, \ell_{2} \geqslant 1$. Since $w_{r_{1}}$ must normalise $Q$ and there is an element $b_{1,2} w \in Q$, with $w \in W_{r_{1}}$, we also have $\left[w_{r_{1}}, b_{1,2} w\right] \in Q$. Therefore $w_{r_{1}-p^{n-2}} \in$ $\Omega_{1}(Q)$, by Lemma 2 and the argument used to establish (46). Thus

$$
i \geqslant r_{1}-p^{n-2}=p^{n-2}(p-2)-1 .
$$

When $p \geqslant 5$, then it follows that

$$
i>p^{n-2} \text {. }
$$

Assume for the moment that (70) is true even for $p=3$. Let $g=b_{1, n-1} x_{n, 2}$ (= $x_{n, 2} b_{1, n-1}$, by Lemma 1 (i)) and put $K=\langle g\rangle$. Consider the subgroup $Q K$. By (70)

$$
\Omega_{1}(Q K) \geqslant \Omega_{1}(Q) \geqslant W_{p^{n-2}} .
$$

Also $g$ must normalise $\Omega_{1}(Q K)$ and hence

$$
\left[w_{p^{n-2}}, g\right]=\left[w_{p^{n-2}}, b_{1, n-1}\right] x_{n, 1} \in \Omega_{1}(Q K) .
$$

Then since $\left[w_{p^{n-2}}, b_{1, n-1}\right] \in \Omega_{1}(Q)$, we have

$$
x_{n, 1} \in \Omega_{1}(Q K) .
$$

Thus $x_{n, 1}=u v$, with $u \in Q, v \in K$, and clearly we must have

$$
v=g^{p(1+\lambda p)}=b_{1, n-1}^{p+\lambda p^{2}} x_{n, 1},
$$

for some integer $\lambda$. Therefore

$$
x_{n, 1}=u b_{1, n-1}^{p+\lambda p^{2}} x_{n, 1},
$$

and so $b_{1, n-1}^{p+\lambda p^{2}}=u^{-1} \in Q$ of exponent $p^{2}$. Since $b_{1, n-1}^{p+\lambda p^{2}}$ has order $p^{n-2}\left(\geqslant p^{3}\right)$, we have a contradiction. Thus $Q$ cannot exist.

It remains to prove that (70) holds for $p=3$. Indeed we shall prove that

$$
i \geqslant r_{1}-p^{n-4}(p-1)-p^{n-3}(p-1),
$$

for all odd $p$. Then

$$
i \geqslant p^{n-1}-2 p^{n-2}+p^{n-4}-1=p^{n-2}\left(p-2+1 / p^{2}-1 / p^{n-2}\right)>p^{n-2}
$$

for all odd $p$ and so (70) is true. 
In order to prove (71), we take $g=w_{r_{1}}^{s} b_{1,4}^{-1} x_{n, 3}$, where $p$ does not divide $s$. Observe that $b_{1,4}$ and $x_{n, 3}$ commute, by Lemma 1 (i). Also $w_{r_{1}}$ and $x_{n, 3}$ commute modulo $W_{r_{1}-p^{n-3}} X_{n, 1}$. Thus working in $H_{n}$ modulo $W_{r_{1}-p^{n-3}}$, we see (from Lemma 2) that

$$
g^{p}=w^{\prime} b_{1,4}^{-p} x_{n, 2}
$$

where $w^{\prime} \in W_{j}$, for $j=r_{1}-p^{n-4}(p-1)$, but $w^{\prime} \notin W_{j-1}$, again by the argument used to prove (46). Similarly

$$
g^{p^{2}}=w^{\prime \prime} b_{1,4}^{-p^{2}} x_{n, 1},
$$

where $w^{\prime \prime} \in W_{k}$, for $k=j-p^{n-3}(p-1)$, but $w^{\prime \prime} \notin W_{k-1}$. Here we are using

$$
b_{1,4}^{-p} \equiv b_{1,3}^{-1} \bmod \Omega_{2}\left(C_{2}\right),
$$

by (32) etc. Since, in the same way, we have

$$
b_{1,4}^{-p^{2}} \equiv b_{1,2}^{-1} \bmod \Omega_{1}\left(C_{2}\right),
$$

we may write

$$
g^{p^{2}}=w^{\prime \prime \prime} b_{1,2}^{-1} x_{n, 1}
$$

for $w^{\prime \prime \prime} \in W_{k} \backslash W_{k-1}$. Recalling that there is an element $b_{1,2} w \in Q\left(w \in W_{r_{1}}\right)$ and putting $K=\langle g\rangle$, it follows that

$$
w^{\prime \prime \prime} w x_{n, 1}=g^{p^{2}} b_{1,2} w \in \Omega_{1}(Q K) .
$$

Also we can choose $s$ such that

$$
w^{\prime \prime \prime} w \notin W_{k-1} .
$$

However, $p^{n-2}(p-1) \geqslant 2 p^{n-2} \Rightarrow i \geqslant r_{1}-p^{n-2}$ (by (69))

$$
\geqslant p^{n-2}-1>p^{n-3} \Rightarrow w_{p^{n-3}} \in Q .
$$

Thus $\left[w_{p^{n-3}}, g\right]=\left[w_{p^{n-3}}, b_{1,4}^{-1}\right] x_{n, 1}$ implies $x_{n, 1} \in \Omega_{1}(Q K)$. Now by (28) and Corollary 1,

$$
g^{p^{3}}=b_{1,2}^{-p}=w_{1}^{-1} \in \Omega_{1}(Q),
$$

and so $\Omega_{1}(Q K)=\Omega_{1}(Q) \times X_{n, 1}$. Then from (72) we have

$$
w^{\prime \prime \prime} w \in \Omega_{1}(Q K) \cap H_{n}=\Omega_{1}(Q) .
$$

Hence $i \geqslant k$ by (73), i.e. we have proved (71). This completes the proof of Theorem 5 . 
Concluding remarks. When we embarked on this work, we were interested to know if every quasinormal subgroup of a finite $p$-group was a term of a composition series consisting of quasinormal subgroups. Now it is difficult to imagine how that supposition could have been further from the truth. So we finish with the following question. Is there a non-trivial subgroup-theoretic property $\mathfrak{X}$ (see [8], page 9 ) of finite $p$-groups such that

(i) $\mathfrak{X}$ is invariant under subgroup lattice isomorphisms, and

(ii) every chain of $\mathfrak{X}$-subgroups of a finite $p$-group can be refined to a composition series of $\mathfrak{X}$-subgroups?

\section{REFERENCES}

[1] T. R. Berger - F. Gross, A universal example of a core-free permutable subgroup, Rocky Mountain J. Math., 12 (1982), pp. 345-365.

[2] J. Cossey - S. E. STONEHEwER, Abelian quasinormal subgroups of finite pgroups, J. Algebra, 326 (2011), pp. 113-121.

[3] M. Hall, The Theory of Groups, Macmillan, New York, 1959.

[4] B. Huppert - N. Blackburn, Finite Groups II, Springer-Verlag, BerlinHeidelberg-New York, 1982.

[5] R. MAIER - P. SCHMID, The embedding of quasinormal subgroups in finite groups, Math. Z., 131 (1973), pp. 269-272.

[6] O. ORE, Structures and group theory I, Duke Math. J., 3 (1937), pp. 149-173.

[7] O. ORE, On the application of structure theory to groups, Bull. Amer. Math. Soc., 44 (1938), pp. 801-806.

[8] D. J. S. RobInson, Finiteness Conditions and Generalized Soluble Groups I, Springer-Verlag, Berlin-Heidelberg-New York, 1972.

[9] S. E. Stonehewer, Permutable subgroups of some finite permutation groups, Proc. London Math. Soc., 28 (3) (1974), pp. 222-236.

[10] S. E. StONEHEWER - G. ZACHER, Abelian quasinormal subgroups of groups, Rend. Mat. Acc. Lincei, 15 (9) (2004), pp. 69-79.

Manoscritto pervenuto in redazione il 12 luglio 2010. 
